\title{
OTIMIZAÇÃO NÃO-LINEAR NA SOLUÇÃO DO PROBLEMA INVERSO DA IRRIGAÇÃO POR SULCOS EM TEMPO REAL
}

\author{
Carlos Alberto Vieira de Azevedo ${ }^{1}$, Gary P. Merkley² e Wynn R. Walker ${ }^{2}$
}

\begin{abstract}
RESUMO
Um modelo matemático computacional de olimização não-linear foi desenvolvido para interagir com um modelo hidráulico de ondas cinemáticas, na obtenção dos parâmetros de Kostiakov-Lewis, a partir do avanço medido (problema inverso da irrigação por sulcos). Este modelo faz parte de um programa computacional, denominado SIRTOM (Surface Irrigation Real-Time Optimization Model), desenvolvido por Azevedo (1992) para solucionar o problema inverso. O modelo foi testado para diferentes condições de campo e as análises demonstraram que, mesmo na presença de vales estreitos e profundos, formados por mínimos locais, o modelo de otimização mostrou-se capaz de sair desses vales e alcançar as regiões mais baixas, onde se encontrava o mínimo global. O modelo exibiu, também, um caminho racional na busca de soluçôes aceitáveis. Verificou-se, ainda, que ele demonstrou possuir habilidade matemática para identificar e corrigir imprecisões em medições de campo dos coeficientes de infiltração; além do mais, forneceu soluções aceitáveis em tempo hábil, o que é fundamental para o programa SIRTOM para se fazer um diagnóstico e controle das condições hidráulicas de um evento de irrigação, visando melhorar o desempenho do sistema de irrigação por sulcos em tempo real.
\end{abstract}

Palavras-chave: infiltração, avanço, desempenho, manejo de irrigação

\section{NONLINEAR OPTIMIZATION IN THE REAL-TIME SOLUTION OF THE INVERSE FURROW IRRIGATION PROBLEM}

\begin{abstract}
A computer nonlinear optimization model was developed to interact upon a kinematicwave hydraulic model to obtain the Kostiakov-Lewis parameters from measured advance data (furrow irrigation inverse problem). This model is part of a computer program, named SIRTOM (Surface Irrigation Real-Time Optimization Model), developed by Azevedo (1992) for solving the inverse problem. The model was tested for different field conditions. The analysis showed that even in the presence of narrow and steep valleys formed by local minima, the optimization model was able to exit these valleys and reach the lower regions, where was the global minimum. Also, the model exhibited a rational convergence path in searching for reliable solutions to the inverse problem, and showed to have a mathematical ability to identify and correct inaccuracies in field measured infiltration coefficients. Besides that, the model provided reliable solutions early enough during the advance phase, which is fundamental to the SIRTOM program for making a real-time diagnosis and control of the irrigation event hydraulic conditions, in order to improve the irrigation system performance.
\end{abstract}

Kcy words: infiltration, advance, performance, irrigation management

\footnotetext{
1 Professor, PhD, Departamento de Engenharia Agrícola, Universidade Federal da Paraíba, Av. Aprigio Veloso 882, Bodocongó, CEP 58109-970, Campina Grande, PB, Brasil, e-mail: cazevedo@deag.ufpb.br, Fone: (083) 310 1318, Fax: (083) 3101184

2 Professor, PhD, Department of Biological and Irrigation Engineering, Utah State University, Logan, Utah, 84322- 4105, US A, email: merkley@cc.usu.edu, Phone: (801) 797 2785, Fax: (801) 7971248.
} 


\section{INTRODUÇÃO}

O problema inverso da irrigação superficial de que trata Azevedo (1992) pode ser resolvido de duas maneiras diferentes: soluções algébricas para os parâmetros de campo podem ser desenvolvidas através de hipóteses que simpli ficam as equações de Saint-Venant (Smerdon et al., 1988; Clemmens, 1991); a segunda alternativa é procurar, via otimização matemática, valores para esses parâmetros, principalmente para a inliltração, que resultem num ajuste adequado do avanço simulado ao medido. Pesquisadores como Elliot et al., (1982), Walker \& Busman (1990), Katopodes (1990), Katopodes et al. (1990), Bautista \& Wallender (1991) e Azevedo (1992) escolheram por esta última alternativa.

A solução do problema inverso da irrigação superficial tem aumentado substancialmente o potencial para desenvolvimento de ferramentas para diagnóstico e controle das condições hidráulicas de um evento de irrigação, objetivandose manter o desempenho do sistema de irrigação num nível satisfatório (Elliotl et al., 1982; Walker \& Busman, 1990). Pesquisadores como Katopodes (1990), Katopodes et al. (1990), Clemmens (1991), e Bautista \& Wallender (1991) têm postulado a necessidade de pesquisas no desenvolvimento de programas computacionais que propiciem uma solução para o problema inverso, mais precisa e obtida em tempo hábil.

Este estudo é parte de uma pesquisa que pretendeu desenvolver um programa computacional de diagnóstico e controle da irrigação por sulcos, como ferramenta para a tomada de decisão na melhoria, em tempo real, do desempenho de um sistema de irrigação. O objetivo desta pesquisa foi desenvolver um modelo matemático computacional de otimização não-linear que, associado a um modelo hidráulico de ondas cinemáticas, pudesse formular e resolver o problema inverso da irrigação por sulcos.

\section{DESENVOLVIMENTO TEÓRICO DO MODELO}

\section{O Método de Powell}

A versão modificada do mélodo de Powell, apresentada por Press et al. (1988) foi adaptada nesta pesquisa para resolver o problema inverso da irrigação por sulcos.

O método de Powell é uma lécnica de minimização multidimensional que se constitui no protótipo da maioria dos métodos direcionais de otimização não-linear; ele determina e especifica o conjunto de vetores que estipulam as direções nas quais o mínimo ótimo deve ser procurado. Este conjunto de vetores inclui vetores unitários como membros. Usando-se uma técnica de minimização unilateral e começando a partir de um ponto correspondente a uma solução inicial, movimentos são feitos ao longo da primeira direção até o seu ponto mínimo, a partir do qual a solução é investigada ao longo da segunda direção até se encontrar o mínimo nela, e assim por diante. $O$ ciclo de iterações sobre o conjunto de direções é repetido até que não se possa fazer outros movimentos, o que significa terse atingido as regiões mais baixas do vale de mínima, indicando, por conseguinte, que a função objetiva tem sido completamente minimizada.

Os vetores direcionais podem ser, eventualmente, atualizados no final de cada ileração. Existem situações numa otimização multidimensional em que a segunda derivada da função numa direção, pode ser bem maior que em outras. Quando isto ocorre, ciclos repetidos sobre todos os $\mathrm{N}$ vetores unitários são necessários para se conseguir algum progresso (muitas vezes insignificante) na busca do ponto ótimo; assim sendo, torna-se necessário obter-se outros componentes para o conjunto de vetores direcionais, mais eficientes que as direções ortogonais.

A seguir, será apresentada uma descrição detalhada do método de Powell. Partes desta descrição foram extraídas de Press et al. (1988) e Acton (1970). O processo de minimização inicia-se procurando-se pela solução mínima ao longo de cada direção ortogonal, variando, então, apenas um parâmetro de infiltração por etapa. Os resultados de uma iteração anterior são usados para melhorar a eficiência da investigação durante a próxima iteração. Se algumas condições preestabelecidas são satisfeitas, a direção anterior, ao longo da qual a função objetiva teve seu maior decréscimo, é descarlada em favor de uma nova direção.

O método modificado de Powell, implementado nesta pesquisa, envolve três elapas: a primeira consiste em identificar o mínimo e sua posição correspondente a cada direção ortogonal; por exemplo, suponha-se que a posição inicial da solução, envolvendo os $\mathbf{N}$ pontos ortogonais, seja descrita pelo vetor $\mathbf{P}_{0}=\left(\mathbf{P}_{1}, \ldots, \mathbf{P}_{\mathbf{n}}\right)$. A função objetiva $(\mathbf{F})$ é escrita da seguinte forma:

$$
\min f\left(P_{n}\right)
$$

onde $\mathbf{P}_{\mathbf{n}}$ é um novo ponto, ao longo do vetor, dado por:

$$
P_{n}=P_{o}+\lambda \xi
$$

sendo $\lambda$ uma distância ao longo do presente vetor fornecida pelo usuário e $\xi$ a respectiva direção. A transversalidade nas $\mathbf{n}$ direções permile reiniciar o processo de busca a partir do mínimo atual e proceder no sentido declivoso, observando-se a quantidade em que $\mathbf{F}$ decresce em cada etapa para identificar o estágio no qual o decréscimo é mais acentuado.

Depois de se minimizar em todas as $\mathbf{n}$ direções atuais, ou seja, na conclusão de uma iteração, dois testes são conduzidos para verilicar se o atual conjunto de direções deve ou não manterse inlaclo para a próxima iteração. Essses testes são baseados nas seguintes avaliações da função-objetivo:

$$
\begin{gathered}
f_{o}=f\left(P_{o}\right) \\
f_{n}=f\left(P_{n}\right) \\
f_{e}=f\left(P_{e}\right) \\
\Delta f
\end{gathered}
$$

cujos pontos $\mathbf{P}_{\mathrm{o}}$ e $\mathbf{P}_{\mathrm{n}}$ correspondem, respectivamente, às posições inicial e final, e $\mathbf{P}_{\mathbf{c}}^{\mathbf{n}}$ é um ponto extrapolado ao longo da nova direção proposta, dado por: 


$$
P_{e}=2 P_{n}-P_{o}
$$

Obviamente, $\mathbf{P}_{\mathrm{e}}$ é um ponto colinear com os dois primeiros pontos numa distância mais abaixo na linha de conexão; portanto, $\mathbf{F}$ é avaliado em três pontos equiidistantes; finalmente, $\mathbf{F}$ é a grandeza absoluta do maior decréscimo na função ocomido ao longo de uma direção particular durante a iteração anterior.

A segunda etapa consiste na realização de dois testes nos valores inicial e revisado da função objetiva:

$$
f_{e} \geq f_{o}
$$

$$
2\left[f_{o}-2 f_{n}+f_{e}\right]\left[f_{o}-f_{n}-\Delta f\right]^{2} \geq \Delta f\left[f_{o}-f_{e}\right]^{2}
$$

Caso uma das condições expressas pelas equações 8 e 9 seja satisfeita, o conjunto atual de direções é mantido e uma nova iteração é processada, fazendo-se $\mathbf{P}_{\mathbf{n}}$ igual a $\mathbf{P}_{v}$. A condição expressa na equação 8 indica que as condições translacional e rotacional, usadas durante a iteração anterior, estão absolutamente exauridas, ou seja, as mesmas já proveram o máximo grau de minimização de que são capazes; portanto, tornase imprescindível incluir uma nova direção. A condição na equação 9 implica em que o maior decréscimo não foi uma contribuição principal da direção na qual o mesmo ocorreu. Especificamente, esse decréscimo pode ter acontecido, na referida direção, devido às contribuições indiretas de outras direções. Outra implicação é que a seção transversal, na direção média, apresenta excelente curvatura, significando que a segunda, derivada ao longo desta direção, é de uma grandeza significante. Neste caso, $\mathbf{f}_{\mathbf{n}}$ está novamente próximo do ponto ótimo e, conseqüentemente, a investigação em busca do mínimo nessa direção tem sido substancialmente explorada.

A terceira elapa consiste em se verificar se os dois testes, efetuados na segunda etapa, são satisfeitos, indicando que a direção média é benéfica devendo, então, substituir a direção na qual ocorreu o maior decréscimo.

\section{O Método de Brent}

O método de Brent (1973) para minimização unidimensional sem a utilização de derivadas foi adaptado a esta pesquisa, para satisfazer os requerimentos do método de Powell por uma técnica unilateral, como parte de seu processo de investigação do mínimo.

Este método é o mais recomendado para ser implementado num algoritmo multidimensional, principalmente quando o cálculo de derivadas é indesejável (Press et al., 1988). O método é uma combinação de dois procedimentos de investigação do mínimo, quais sejam: as técnicas de interpolação seccional (golden-section) e de interpolação parabólica inversa. O método encontra o mínimo de uma função f que foi inicialmente delinido no intervalo [a, b]. A princípio, a lunção f não é avaliada nos pontos extremos a e $\mathbf{b}$, a não ser que eles estejam bastante próximos. Num determinado caso em que o mínimo esteja em a ou em b, um ponto interior, localizado a uma distância $\mathbf{2 ( t o l ) ~ d e ~}$ a ou de b, é gerado, sendo tol a tolerância descrita abaixo.

Uma etapa típica do procedimento trabalha com seis pontos (a, b, u, v, w e x) onde nem todos eles são distintos.
Embora a posição desses pontos mude durante o processo de minimização, a omissão de subscritos neles não deve causar confusão. Inicialmente, os pontos $\mathbf{v}$, w e $\mathbf{x}$ são definidos como:

$$
v=w=x=a+\left(\frac{3-\sqrt{5}}{2}\right)(b-a)
$$

em que o número $\left(3-5^{0,5}\right) / 2=0,381966$ é escolhido arbitrariamente, tornando a primeira elapa de cálculo tal como uma busca por interpolação seccional (golden-section). No início de uma iteração, os pontos $\mathbf{a}, \mathbf{b}, \mathbf{u}, \mathbf{v}, \mathbf{w}$ e $\mathbf{x}$ sempre têm a seguinte conotação: os pontos a e b definem o intervalo em que o mínimo se encontra; $\mathbf{x}$ é o ponto correspondente ao menor valor atual de $\mathbf{f} ; \mathbf{w}$ é o ponto que fornece o próximo menor valor de $\mathbf{f} ; \mathbf{v}$ guarda o valor anterior de $\mathbf{w}$; e u é o último ponto no qual f foi avaliado.

A tolerância tol é a associação de uma tolerância relativa e com uma tolerância absoluta $\mathbf{t}$. Tem-se, então:

$$
\text { tol }=\varepsilon|x|+t
$$

A Equação 11 indica que o ponto $\mathbf{x}$ aproxima um mínimo a uma precisão de $\mathbf{2}(\mathbf{t o l})+\mathrm{d}<\mathbf{3}(\mathbf{t o l})$ e dé a distância a partir do mínimo. Os valores posilivos para e e $\mathbf{t}$ são fornecidos pelo usuário da técnica. Valores razoáveis para e estão numa ordem de grandeza de $\hat{I}^{1.5}$ ou maior, e Î é a precisão do computador.

Uma descrição matemática do método de Brent pode ser feita tal como segue: permite $\mathbf{m}=\mathbf{( 0 . 5}(\mathbf{a}+\mathbf{b})$ ser o ponto intermediário do intervalo que contém o mínimo. Quando |x$\mathbf{m} \mid £ 2$ (tol)-0.5(b-a) o processo de busca é encerrado, e $\mathbf{x}$ é considerado a posição aproximada do mínimo. Até que essa condição seja alcançada, valores para $\mathbf{p}$ e $\mathbf{q}(\mathbf{q}>\mathbf{0})$ são calculados de maneira que $\mathbf{x}+\mathbf{p} / \mathbf{q}$ será o ponto de curvatura da parábola que passa pelos pontos $\left(\mathbf{v}, \mathbf{f}_{\mathbf{v}}\right),\left(\mathbf{w}, \mathbf{f}_{\mathbf{w}}\right)$ e $\left(\mathbf{x}, \mathbf{f}_{\mathbf{x}}\right)$. No caso de dois ou mais desses pontos coincidirem, ou mesmo se a parábola degenerar para uma linha reta, $\mathbf{q}=\mathbf{0}$.

Os pontos $\mathbf{p}$ e $\mathbf{q}$ são dados por:

$$
\begin{aligned}
& p= \pm\left\{(x-v)^{2}\left[\int_{x}-\int_{w}\right]-(x-w)^{2}\left[\int_{x}-\int_{v}\right]\right\} \\
& p= \pm(x-v)(x-w)(w-v)^{\prime}\left\{(x-w) \int[v, w, x]+\int[w]\right\} \\
& q= \pm 2\left\{(x-v)\left[f_{x}-f_{w}\right]-(x-w)\left[f_{x}-f_{v}\right]\right\} \\
& q= \pm 2(x-v)(x-w)(w-v) \int[v, w, x]
\end{aligned}
$$

Os efeitos dos erros de arredondamento nos cálculos de $\mathbf{p}$ e $\mathbf{q}$ são minimizados quando x se encontra próximo de um mínimo e quando a segunda derivada é posiliva.

Faça e igual ao valor de $\mathbf{p} / \mathbf{q}$ no penúltimo ciclo. Se $|\mathbf{e}| £$ tol, $\mathbf{q}=\mathbf{0}, \mathbf{x}+\mathbf{p} / \mathbf{q}$ Ï $(\mathbf{a}, \mathbf{b})$, ou $|\mathbf{p} / \mathbf{q}|^{3} \mathbf{( 0 . 5}|\mathbf{e}|$, então uma etapa de interpolação seccional é realizada; assim sendo, o próximo valor para u é dado por:

$$
u=\left\{\begin{array}{l}
\left(\frac{\sqrt{5}-1}{2}\right) x+\left(\frac{3-\sqrt{5}}{2}\right) a \rightarrow x \geq m, \\
\left(\frac{\sqrt{5}-1}{2}\right) x+\left(\frac{3-\sqrt{5}}{2}\right) b \rightarrow x<m
\end{array}\right\}
$$


Se as próximas $\mathbf{k}$ elapas são interpolações seccionais atinge-se, então, o limite da escolha ótima na medida em que $\mathbf{k} \rightarrow \infty$; caso contrário, uma etapa de interpolação parabólica é realizada, isto é, $\mathbf{u}$ é considerado igual a $\mathbf{x}+\mathbf{p} / \mathbf{q}$, com a ressalva de que as distâncias $\mid \mathbf{u}$ - x|, u - a e b - u devem, necessariamente, ser pelo menos iguais a tol; em seguida, fé avaliada no novo ponto $\mathbf{u}$, os pontos $\mathbf{a}, \mathbf{b}, \mathbf{v}, \mathbf{w}$ e $\mathbf{x}$ são alualizados e o ciclo é repetido. $\mathrm{O}$ procedimento nunca avalia $\mathbf{f}$ em dois pontos que estejam próximos, além da distância tol, resultando em $\delta$ unimodal para algum $\delta<$ tol, o que é suficiente para garantir que o mínimo global seja encontrado com uma precisão de $\mathbf{2}(\mathbf{t} \mathbf{l})+\delta$.

$\mathrm{O}$ procedimento encerra-se quando $\mathbf{x}=\mathbf{b}$-tol (ou, simetricamente, a + tol) depois que uma etapa de interpolação parabólica tenha sido realizada com a condição $|\mathbf{u}-\mathbf{x}| \geq \mathbf{t o l}$ satisfeita. O próximo ponto da interpolação parabólica encontrase bastante próximo de $\mathbf{x}$ e $\mathbf{b}$; então, $\mathbf{u}$ é forçado a ser igual a $\mathbf{x}$ - tol. Se $\mathbf{f}(\mathbf{u})>\mathbf{f}(\mathbf{x})$, então num deslocamento para $\mathbf{u}(\mathbf{b}$ - a) será 2(tol) satisfazendo, assim, ao critério de término.

\section{O Método do Intervalo de Mínima}

O procedimento do intervalo de mínima apresentado por Press et al. (1988) foi adaptado nesta pesquisa para preencher o requerimento do método de Brent, no que concerne a um intervalo que delimita o mínimo. O procedimento investiga o intervalo de mínima [a, b] de uma função $\mathbf{f}$ na direção da declividade (downhill direction); mais precisamente, o processo começa com uma tentativa inicial para o intervalo que, a seguir, é aumentado por um fator constante ou por uma extrapolação parabólica de pontos anteriores. Os pontos da esquerda e do meio do intervalo são definidos assim que se inicia a investigação na direção da declividade. Seguindo-se a isto, um incremento suficientemente grande para frear o movimento de descida, determinará o ponto à direita (ponto mais alto).

A seguinte descrição apresenta mais detalhes do procedimento. A solução inicial para a e b é fornecida pelo usuário. Neste estudo, o método dos dois pontos (Elliott \& Walker, 1980) fornece a solução inicial para a e b e é calculado da seguinte forma:

$$
b=a+\lambda \xi B X P
$$

Sendo que_BXP é um fator constante que define o tamanho do intervalo de mínima inicial. A função é avaliada para as soluções iniciais a e b; então, se $\mathbf{f}_{\mathbf{b}}>\mathbf{f}_{\mathfrak{a}}, 0$ procedimento inverte as funções de $\mathbf{a}, \mathbf{b}, \mathbf{f}_{\mathbf{a}}$ e $\mathbf{f}_{\mathbf{b}}$, de maneira que o movimento de descida seja estabelecido na direção de a para b. A solução inicial para o terceiro ponto (c) é dado por:

$$
c=b+G O L D(b-a)
$$

em que GOLD corresponde à fração "golden-section" mais um. Esta constante é a base para definição de sucessivos intervalos. Os cálculos a seguir são repetidos enquanto $\mathbf{f}_{\mathbf{b}}>\mathbf{f}_{\mathbf{c}}$.

Um ponto $\mathbf{u}$, que corresponde a uma extrapolação parabólica a partir dos pontos $\mathbf{a}, \mathbf{b}$ e $\mathbf{c}$, é calculado por:

$$
u=b-0,5\left(\frac{d_{2} q-d_{1} r}{d_{3}}\right)
$$

donde

$$
\begin{aligned}
& d_{1}=b-a . \\
& d_{2}=b-c . \\
& r=d_{1}\left(f_{b}-f_{c}\right) . \\
& q=d_{2}\left(f_{b}-f_{a}\right) \\
& d_{3}=|q-r| .
\end{aligned}
$$

Mas se $\mathrm{q} \leq \mathrm{r}_{1}$

$$
d_{3}=-d_{3}
$$

A extrapolação para u não vai além do limite ulim, dado por:

$$
u \lim =b+U I L(c-b)
$$

sendo $\mathbf{U} \mathbf{I L}$, a grandeza máxima permitida para um incremento num ajuste parabólico. Agora, várias possibilidades são testadas. O parabólico u encontra-se entre b e $\mathbf{c}$, se:

$$
(b-u)(u-c)>0
$$

Se a condição da Eq. 25 é satisfeita, então mais dois testes são feitos para se verificar a existência de um mínimo:

$$
s e \rightarrow f_{u}<f_{c}
$$

existe um mínimo entre a e c; caso contrário,

$$
s e \rightarrow \int_{u}>\int_{b}
$$

o mínimo está entre a e u. Se as condições nas Eqs. 26 e 27 não foram satisfeitas, significa, então, que o ajuste parabólico não foi útil; conseqüentemente, um novo valor para u é calculado por:

$$
u=c+G O L D(c-b)
$$

Se a condição na Eq. 25 não é satisfeita, o ajuste parabólico estará entre c e seu limite permissível, se: 


$$
(c-u)(u-u \lim )>0
$$

Se a condição da Eq. 29 é satisfeita e $\mathbf{f}_{\mathbf{u}}<\mathbf{f}_{\mathrm{c}}$, o procedimento altera as funções de $\mathbf{b}, \mathbf{c}, \mathbf{u}, \mathbf{u}_{\mathrm{m}}, \mathbf{f}_{\mathrm{b}}, \mathbf{f}_{\mathrm{c}}, \mathbf{f}_{\mathrm{u}}$, , $\mathbf{f}_{\mathrm{um}}$ para manter o movimento descendente. $\mathbf{u}_{\mathrm{m}}$ é, então, dado pela Eq. 28. Caso a Eq. 29 não seja satisfeita, o parabólico u será limitado ao seu máximo permissível (ulim) se:

$$
(u-u \lim )(u \lim -c) \geq 0
$$

Em contrário, o ajuste parabólico é rejeitado e um novo valor para u é calculado pela Eq. 28. Uma substituição de posições é feita para eliminar o ponto mais antigo e o processo iterativo é, então, repetido. A configuração final do processo de delimitação do intervalo de mínima apresentar-se-á da seguinte maneira: Os valores das abscissas $\mathbf{a}, \mathbf{b}$ e $\mathbf{c}$ resultam em $\mathbf{b}$ entre $\mathbf{a}$ e $\mathbf{c}$, e $\mathbf{f}_{\mathrm{b}}$ menor que ambos os $\mathbf{f}_{\mathrm{a}}$ e $\mathbf{f}_{\mathbf{c}}$.

\section{RESULTADOS E DISCUSSÃO}

Na validação do modelo matemático computacional de otimização não-linear, desenvolvido nesta pesquisa, utilizaramse dados de avaliação de campo de sistemas de irrigação por sulco, que constam em Azevedo (1992).

A otimização consistiu na minimização da soma absoluta das di ferenças entre o tempo de avanço simulado e o medido em campo (função-objetivo denominada resíduo). Pretendeu-se verificar como se comporta a topografia da superfície da funçãoobjetivo para diferentes condições de campo. Constatou-se que esta função-objetivo apresentou, em alguns casos, uma topografia cooperativa, isto é, suave o suficiente para facilitar significativamente o processo de ajuste; isto mostra que as funções-objetiva, nesses estudos de caso, foram aproximadamente parabólicas próximo do mínimo bastando, para tal, uma simples interpolação parabólica através de três pontos quaisquer para facilmente se localizar o mínimo. A função-

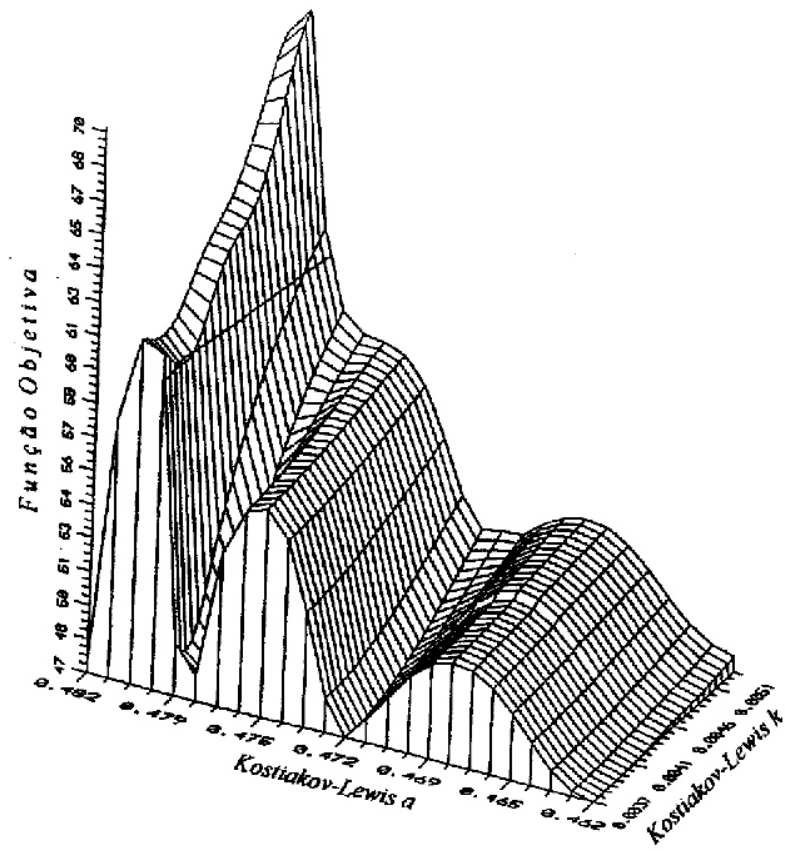

Figura 1. Vale de minima para o sulco Flowell Wheel ajustado-se k e a. objetivo no sulco FWF (Flowell Wheel Furrow, Fig. 1) é tipicamente cooperativa porque exibe uma superfície relativamente regular; no entanto, o sulco KNWF (Kimberly Nonwheel Furrow, Fig. 2) ilustra um bom exemplo de uma função não-cooperativa, uma vez que a superlície é bastante irregular.

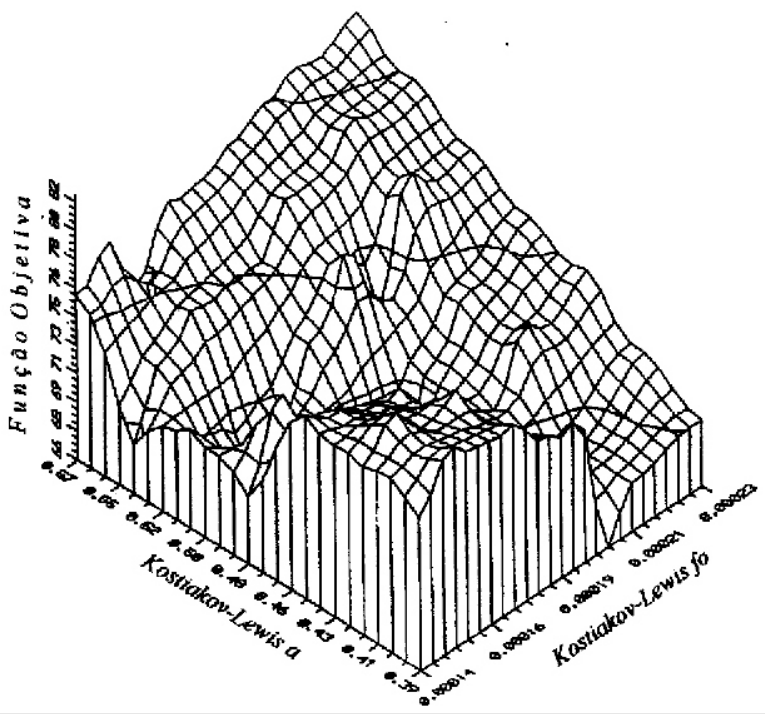

Figura 2. Vale de minima para o sulco Kimberly Nonwheel ajustado-se kea.

Verificou-se que a natureza da função-objetivo foi definida pelo grau de variabilidade espacial em parâmetros de campo e, portanto, uma função não-cooperativa, neste estudo, expressa um alto grau de variabilidade espacial, como no caso do sulco KNWF (Fig. 2).

Um problema que poderá desafiar o algoritmo de olimização não-linear na busca pelo mínimo, é a existência de vales estreitos e profundos formados por mínimos locais; tal situação ocorreu no sulco FWF (Fig. 1). Constata-se que o algoritmo lidou adequadamente com tais siluaçōes (indesejáveis) mostrando que o procedimento de Brent trabalhou com um incremento de distância capaz de promover movimentos, ao longo do vetor direcional, suficientemente longos para sair desses vales; contrariamente, movimentos curtos não permitiriam, ao algoritmo de otimização, alcançar as regiões mais profundas do vale. porque o valor da função-obietivo não mudaria

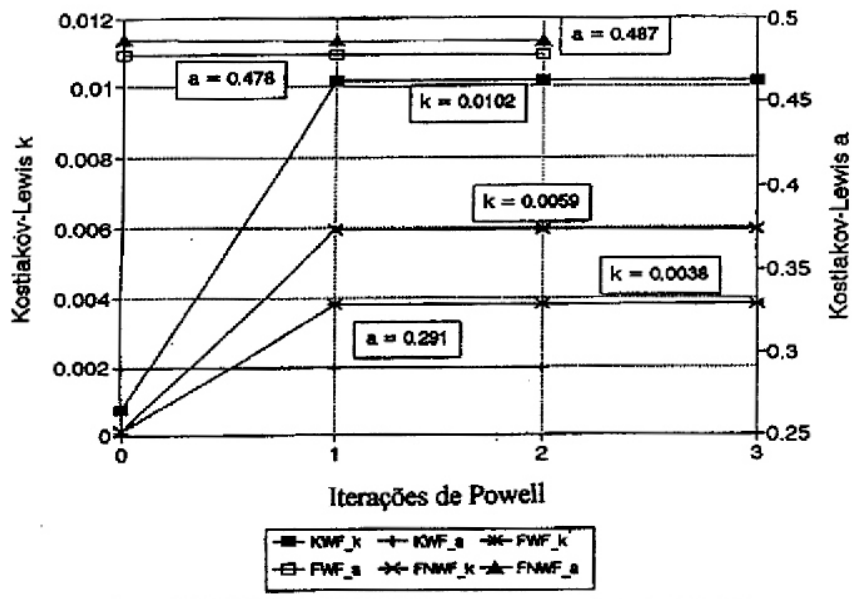

Figura 3. Caminho de convergência do algoritimo ajustando-se $\mathrm{k}$ e a (FWF, FNWF, KWFe KNWF). 
significativamente de uma iteração a outra, causando uma parada no procedimento de Powell.

Para se verificar a performance global do algoritmo de otimização, seu comportamento, investigando a solução inversa ótima, deve necessariamente ser identificado. As Figuras 3 a 5 mostram o caminho de convergência do algoritmo para os sulcos FWF, FNWF, KWF AMALGACQ. Em todos esses sulcos, o algoritmo realizou mudanças inteligentes nos valores dos parâmetros de ajuste. Uma vez que o método dos dois-pontos subestimou os valores de $\mathbf{k}$, a iniciativa do procedimento de Brent, durante todo o processo de sucessivas minimizações, foi corrigir essas subestimativas; em oulras palavras, o algoritmo de otimização não desperdiçou tempo investigando soluções mínimas para a função-objetivo, promovendo decréscimos na solução inicial de k. O mesmo fenômeno ocorreu nos sulcos

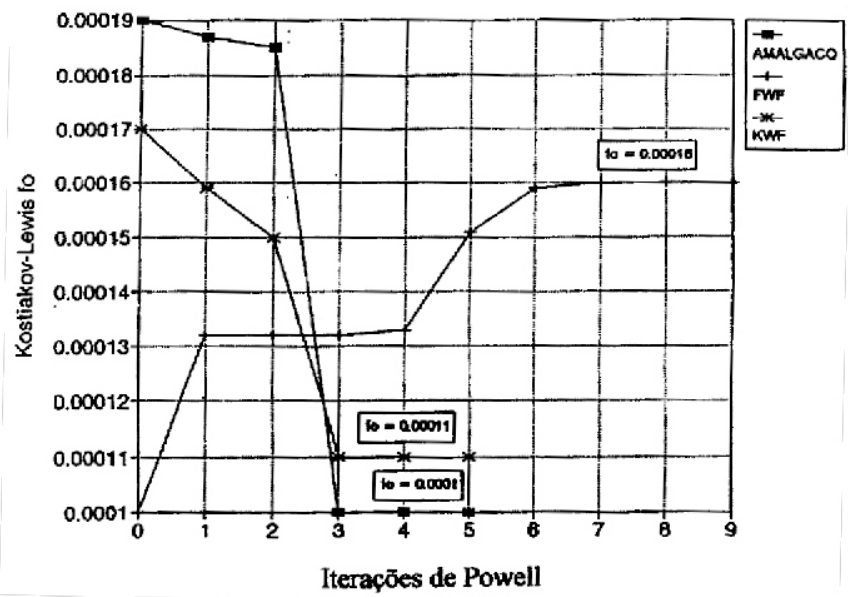

Figura 4. Caminho de convergência do algoritmo ajustando-se dois e três parâmentro (sulco Amalgaç).

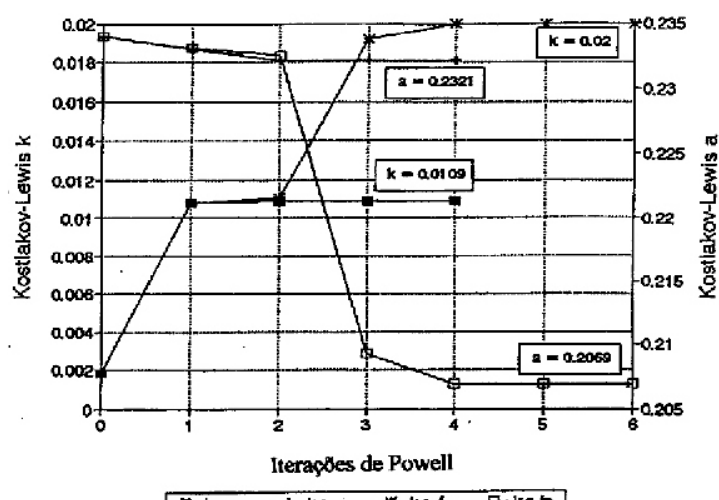

Figura 5. Caminho de convergência do algoritmo ajustando-se dois e três parâmentro (sulcos FWF, KWF c Amalgacq).

KWF e AMALGACQ (Fig. 4) cujos valores medidos de $\mathbf{f}_{\mathbf{n}}$ foram superestimados. Quando o valor de campo de $\mathbf{f}_{o}$ para o sulco FWF foi propositadamente subestimado pelos autores, o algoritmo de otimização ainda manteve comporlamento racional.

Procedimentos de diagnóstico e controle das condições hidráulicas na irrigação por sulco, requerem soluções para o problema inverso oblidas em tempo curlo, de maneira que ajustes, num tempo real, na operação do sistema de irrigação, se tornem possíveis em tempo hábil. A Tabela 1 fornece os tempos requeridos para se solucionar o problema inverso nos sulcos Flowell, Kimberly, Amalga e Guf. Esta tabela compara os tempos de obtenção da solução quando, simultaneamente, se ajustaram os três parâmetros de Kostiakov-Lewis e apenas $\mathbf{k}$ e a, usando-se a fase completa do avanço medido. As colunas $5 \mathrm{e}$ 6 desta tabela correspondem ao ajuste, de uma só vez, da fase de avanço completa, enquanto as colunas 7 e 8 fornecem o tempo total gasto para ajustar, isoladamente, diferentes trechos do avanço simulado, culminando com o ajuste da fase de avanço completa. Nessa última estratégia de ajuste começou-se ajustando o trecho correspondente aos dois primeiros pontos de avanço e, nos ajustes subsequientes, esse trecho era acrescido do segmento referente ao próximo ponto. Essa estratégia simula uma situação de determinação das características de infiltração d'água no solo durante a fase de avanço, situação esta ideal e a que se propôs mais prioritariamente esta pesquisa. Verifica-se que os maiores requerimentos de tempo ocorrem quando a solução inversa é obtida durante a fase de avanço e se ajustam simultaneamente, os três parâmetros de Kostiakov-Lewis. Constata-se que, quanto maior forem o grau de variabilidade

Tabela 1. Comparação entre os tempos requeridos para obtenção da solução inversa, para as condições de campo estudadas

\begin{tabular}{|c|c|c|c|c|c|c|c|}
\hline \multirow{3}{*}{$\begin{array}{l}\text { Dados dc } \\
\text { Campo } \\
\text { Estudados }\end{array}$} & \multirow{3}{*}{$\begin{array}{c}\text { Duraçü } \\
\text { do Avanço } \\
\text { Completu } \\
\text { (min) }\end{array}$} & \multirow{3}{*}{$\begin{array}{l}\text { Núniero } \\
\text { de Pontos } \\
\text { de Ararsó } \\
\text { Ajustados }\end{array}$} & \multirow{3}{*}{$\begin{array}{c}\text { Duraçäo } \\
\text { da } 2^{A} \\
\text { Metade do } \\
\text { Avanço } \\
\text { (min) }\end{array}$} & \multicolumn{2}{|c|}{$\begin{array}{c}\text { Farâmetros Ajustados } \\
\text { (1) }\end{array}$} & \multicolumn{2}{|c|}{$\begin{array}{l}\text { Parâmetros Ajustisdos } \\
\text { (2) }\end{array}$} \\
\hline & & & & $\mathrm{k}$ & $\mathrm{K}$, a, fo & k, a & $\mathrm{K}, \mathrm{a}, \mathrm{fo}_{\mathrm{o}}$ \\
\hline & & & & \multicolumn{2}{|c|}{$\begin{array}{c}\text { Tempo de Solução } \\
\text { (min:sec) (3) }\end{array}$} & \multicolumn{2}{|c|}{$\begin{array}{c}\text { Tempo de Solução } \\
\text { (min:sec) (3) }\end{array}$} \\
\hline GITCQ & 28 & 7 & & 00:01 & $00: 02$ & 00.05 & 0006 \\
\hline GUFIO & 13 & 7 & & $00: 01$ & $00: 02$ & 0004 & 0005 \\
\hline GITPOP & 19 & 7 & & $00: 01$ & $00: 02$ & $00: 03$ & 0004 \\
\hline AMALGADQ & 193 & 13 & & $00: 177$ & $60: 10$ & $01: 08$ & $0: 11$ \\
\hline AMALGACQ & 232 & 13 & 134 & 03:05 & $07: 37$ & $08: 13$ & $16: 01$ \\
\hline AMALGAIQ & 338 & 13 & & $00: 17$ & $00: 19$ & $01: 02$ & 01:04 \\
\hline FWF & 400 & 15 & 359 & 04:89 & 06:00 & $08: 00$ & 13:00 \\
\hline FNWF & 432 & 14 & 372 & $05:[5]$ & $08: 00$ & $16: 00$ & 21:00 \\
\hline KWF & 208 & 7 & 133 & $00: 15$ & $90: 17$ & $02: 00$ & 0300 \\
\hline KNWF & 560 & & 365 & $08:(6)$ & $11: 00$ & $12: 00$ & 1800 \\
\hline
\end{tabular}

Avanço simulado ajustado: (1) de uma só vez e (2) por etapas; (3) tempos baseados num computador DELL $386 / 25 \mathrm{MHz}$

espacial nos parâmetros de campo agregado aos dados medidos de avanço, a quantidade de informações da fase de avanço utilizada no ajuste e o grau de não linearidade na curva de avanço, maior também será o tempo necessário para obtenção da solução inversa. Os tempos de solução estão exibindo uma variabilidade bem maior nos sulcos AMALGACQ, FNWF e KNWF que nos demais. Sabe-se, entretanto, que dentre as condições de campo estudadas nesta pesquisa o sulco KNWF é o que agrega o maior grau de variabilidade; no entanto, mesmo possuindo o maior grau de não linearidade na curva de avanço (tempo de avanço no final da área igual a $560 \mathrm{~min}$ ) ele apresentou um tempo de solução menor que o sulco FNWF. Este fato ocorreu devido ao sulco FNWF possuir uma quantidade de informações sobre a fase de avanço ( 15 pontos) bem maior que o sulco KNWF (7 pontos). Observa-se que o sulco FNWF (sulco não compactado) agrega mais variabilidade espacial que o sulco FWF (sulco compactado) porque as di (erenças entre o grau de não linearidade na curva de avanço e entre a quantidade de informações sobre a fase de avanço utilizada no ajuste, são relativamente pequenas existindo, porém, uma diferença de 8 minutos no tempo de solução. A diferença acentuada entre os tempos de solução nos sulcos KWF (sulco compactado) e KNWF (sulco não compactado) mostra que variabilidades espaciais associadas a avanços mais lentos afetam muito mais o tempo de solução que a quantidade isolada de informaçōes do avanço medido utilizada no ajuste. A quarta coluna da Tabela 1 mostra o tempo que a 
água gastou para se deslocar da metade ao final da área irrigada. Comparações entre esta coluna e a última mostram que os tempos de solução são, essencialmente, pequenos, em alguns casos bastante insignificantes, considerando-se o propósito principal desta pesquisa, que é verificar a possibilidade de se alterar a fase de avanço em tempo hábil. O tempo de solução para o sulco Amalgacq foi apenas de $12 \%$ do tempo requerido para a água avançar da metade da área para seu final. Prognósticos confiáveis para a solução inversa nos sulcos FNWF e KNWF foram leitos, respectivamente, numa quantidade de tempo correspondente a $6 \%$ e $5 \%$ do tempo durante o qual ocorreram estágios adiantados da fase de avanço (melade da área à montante). Esta análise mostra que o programa SIRTOM é capaz de prognosticar o desempenho do sistema de irrigação, para os estudos de caso desenvolvidos nesta pesquisa, bem antes do lérmino da fase de avanço, mesmo porque o tempo de solução discutido neste texto refere-se à obtenção da solução, ajustandose a fase completa de avanço; no entanto, acredita-se que, na maioria dos casos, haverá a necessidade de se ajustar apenas a primeira melade da porção do avanço (ou um pouco mais além disso) para lins de diagnóstico e controle das condições hidráulicas de um evento de irrigação; assim sendo, o tempo de solução será, então, bem menor porque, além de se reduzir a quantidade de informações utilizadas no ajuste, muito pouca não-linearidade existe nesse estágio da fase de avanço.

Aparentemente, os procedimentos de otimização nãolinear utilizados nesta pesquisa são bastante eficazes às complexas condições do problema inverso da irrigação por sulcos. Para a maioria dos sulcos estudados, as soluções inversas aceiláveis para $\mathbf{k}$ e a foram encontradas com a realização de apenas uma iteração em Powell, mas $\mathbf{f}_{\text {o }}$ requereu de 3 a 4 iterações para a obtenção da solução ótima.

\section{CONCLUSÕES}

1. Dependendo do grau de variabilidade espacial em parâmetros de campo, a topogralia da função-objetivo pode ser tanto cooperativa como não-cooperativa.

2. Na presença de vales estreitos e profundos, formados por mínimos locais, o algoritmo de olimização não-linear mostrou-se capaz de sair desses vales e alcançar regiões mais baixas, onde se encontrava o mínimo global.

3. O algoritmo de otimização não-linear exibiu um caminho racional na busca de soluções inversas aceiláveis alterando, de forma inteligente, os parâmetros de KostiakovLewis.
4. O algoritmo de otimização não-linear forneceu soluções inversas aceitáveis em tempo hábil, o que é lundamental para um programa computacional de diagnóstico e controle que visa melhorar o desempenho de um sistema de irrigação em tempo real.

\section{REFERÊNCIAS BIBLIOGRÁFICAS}

ACTON, F.S. Numerical methods that work. 1.ed. New York: Harper and Row, 1970. 235p.

AZEVEDO, C.A.V. de. Real-time solution of the inverse furrow advance problem. Logan: USU, 1992. 275p. Tese de Doutorado.

BAUTISTA, E., WALLENDER, W.W. Spatial variability of infiltration in furrows. Transactions of the $\mathbf{A S} \mathbf{\Lambda} \mathbf{E}$, St. Joseph , v. 28 , n. 3, p. $1846-1851,1985$.

CLEMMENS, A.J. Direct solution to surface irrigation advance inverse problem. Journal of Irrigation and Drainage Engineering, ASCE, New York, v. 117, n. 4, p. 578-594, 1991.

ELLIOTT, R.L., WALKER, W.R. Furrow irrigation infiltration and advance functions. Transactions of the ASAE, St. Joseph, v. 25, n. 2, p. 396-400, 1980.

ELLIOTT, R.L., WALKER, W.R., SKOGERBOE G.V. Zeroinertia modeling of furrow irrigation advance. Journal of Irrigation and Drainage Engineering, ASCE, New York, v. 108, n. IR3, p. 179-195, 1982.

KATOPODES, N.D. Observability of surface irrigation advance. Journal of Irrigation and Drainage Engineering, ASCE, New York, v. 116, n. 5, p. 656-674.30, 1990.

KATOPODES, N.D., STRELKOFF, T. Hydrodynamics of border irrigation-complete model. Journal of Irrigation and Drainage Enginecring, ASCE, New York, v. 103, n. IR3, p. 309-324, 1977.

PRESS, W.H., FLANNERY, B.P., TEUKOLSKY, S.A., VETTERLING, W.T. Numerical recipes in C: the art of scientific computing. 1.ed. Cambridge: Cambridge University Press, 1988. 735p.

SHERMAN, B., SINGH, V.P. A kinematic model for surface irrigation: an extension. Water Resources Research, Phoenix, v. 18, n. 3, p. 659-667, 1982.

WALKER, W.R., BUSMAN, J.D. Real-time estimation of furrow infiltration. Journal of the Irrigation and Drainage Engincering, ASCE, New York, v. 116, n. 3, p. 299-318, 1990. 